\title{
Quantum reference frames and deformed symmetries
}

\author{
Florian Girelli ${ }^{1, *}$ and David Poulin ${ }^{2,+}$ \\ ${ }^{1}$ SISSA, Via Beirut 2-4, 34014 Trieste, Italy, \\ and INFN, Sezione di Trieste, Trieste, Italy \\ ${ }^{2}$ Center for the Physics of Information, California Institute of Technology, Pasadena, California 91125, USA
}

(Received 24 November 2007; published 12 May 2008)

\begin{abstract}
In the context of constrained quantum mechanics, reference systems are used to construct relational observables that are invariant under the action of the symmetry group. Upon measurement of a relational observable, the reference system undergoes an unavoidable measurement "back-action" that modifies its properties. In a quantum-gravitational setting, it has been argued that such a back-action may produce effects that are described at an effective level as a form of deformed (or doubly) special relativity. We examine this possibility using a simple constrained system that has been extensively studied in the context of quantum information. While our conclusions support the idea of a symmetry deformation, they also reveal a host of other effects that may be relevant to the context of quantum gravity, and could potentially conceal the symmetry deformation.
\end{abstract}

DOI: 10.1103/PhysRevD.77.104012

PACS numbers: 04.50.Kd, 03.67.- a, 04.60. $-\mathrm{m}$

\section{INTRODUCTION}

A common problem faced by all approaches to quantum gravity (QG) is the derivation of a semiclassical theory. There are two aspects to this problem. On the one hand, the theory must predict a flat space-time in a well-defined limiting regime. On the other hand, the leading corrections of the theory in this flat space-time approximation need to be well understood and characterized. These corrections are crucial as they establish a connection to observations, either e.g. in astrophysical phenomenon [1] or in particle accelerators [2].

The problem of deriving a low-energy limit is not specific to QG but rather common in many other areas of physics. In many cases, constructing an ad hoc effective low-energy theory rather than deriving it from a more fundamental one has been quite successful. In condensed matter physics for instance, phenomenological theories can vary accurately describe exotic phases of matter, e.g. [3], which are otherwise difficult to understand from a more fundamental standpoint. What distinguishes QG is of course the rarity of empirical data. Nonetheless, general principles and physical intuition can guide the construction of such effective theories, and their physical prediction can be confronted with more fundamental QG theories and available experimental data.

An area of research that goes along these lines is deformed special relativity (DSR) [4-7]. Since flat spacetime is characterized by its symmetries-namely the Poincare group - one can modify these symmetries in order to describe potential QG corrections. This approach has the advantage of preserving the relativity principle, albeit through a nonlinear realization of the symmetries. The

\footnotetext{
*girelli@sissa.it

+dpoulin@ist.caltech.edu
}

consequences of DSR are not yet fully understood and the theory still undergoes some important developments.

Recently, it has been suggested that DSR may be derived as an effective theory of measurement in a fluctuating quantum space-time [8-10]. The key insight is that observables in QG are relational-constructed using physical reference frames (RF). The kinematical coordinates in terms of which the theory is usually formulated are not themselves observable, instead only diffeomorphic invariant relations among them have a direct physical meaning. This aspect of quantum gravity has been discussed at length for many years, see [11-13] for overviews. The idea then is that quantum fluctuations of the RF could lead to an effective deformation of the symmetries of observables, which may be expressible as a form of DSR (see also [14] for a similar argument).

Relational observables are relevant not only to QG, but to any theory endowed with symmetries. Indeed, only the observables that are invariant under the action of the symmetry group - the constrained or Dirac observables-can be probed directly and thus acquire a physical meaning. Quantum relational observables have been discussed quite extensively in the literature [15-20]. Recently, constrained measurements have spurred an interest for quantum RF in the quantum information community, see [19,21-27] for different aspects of the problem. In this setting, the quantum fluctuations experienced by the RF may limit its use to extract information from other systems, thus placing fundamental limitations on quantum communication and computation. These effects have been studied in great detail using exactly solvable models.

In this paper, we build upon this knowledge and examine the possibility of symmetry deformation in a simple constrained model. Our conclusion is that, under some circumstances to be specified, the effect of quantum fluctuations on the RF can be interpreted as a deformation of its 
symmetry group. While the analogy between the model investigated here and QG is far from perfect, we believe that our analysis supports the idea of DSR as an effective low-energy theory. This conclusion is corroborated by some nontrivial properties of many-particle observables common to the two models, which we briefly outline. On the other hand, we also point out a number of other consequences of the quantum fluctuations of the RF-in particular their fundamentally irreversible nature-that were overlooked in the QG context and may require further investigation.

The remainder of this paper is organized as follows. In the next section, we summarize the proposal [8-10] of DSR as an effective theory of measurement on a fluctuating quantum space-time. Section III presents the constrained model to be investigated in this paper and provides a detailed description of the measurement back-action on the RF [27]. Section IV addresses the possibility of experimentally detecting the measurement back-action in this simple model, and places fundamental constraints on the regime where it can be detected. The following section addresses the possibility of extending those conclusions to the context of DSR, and also presents other analogies existing between the two models. Finally, Sec. VI summarizes our conclusions.

\section{DEFORMED SPECIAL RELATIVITY}

Deformed special relativity is motivated by the existence of a universal quantum-gravitational energy scale $M_{P}$, the Plank mass. Imposing a Planckian maximum value to either the energy or the 3-dimensional momentum conflicts with the usual realization of the boost sector of the Lorentz group, which mixes all different length scales. However, a cutoff is consistent with a deformed-or more precisely nonlinear-realization of the Lorentz symmetry. As a consequence, the mass shell relation is modified to

$$
E^{2}=m^{2}+p^{2} \rightarrow E^{2}=m^{2}+p^{2}+\sum_{n=1}^{\infty} \alpha_{n}\left(p, M_{P}\right),
$$

where $\alpha_{n}$ is a function of dimension mass squared, and $p=|\vec{p}|$.

There are various ways to incorporate this deformation, for example, using quantum groups technics [28]. A more pedestrian way consists in directly defining a nonlinear realization of the Lorentz group on momentum space, leaving the space-time construction aside. To do so, one assumes the existence of an auxiliary momentum variable $\pi$ carrying a linear representation of the Lorentz group. This auxiliary momentum is related the physical momentum $p$ via a nonlinear invertible "deformation map":

$$
p=\mathcal{U}_{M_{P}}(\pi) .
$$

The choice of $\mathcal{U}_{M_{P}}$ is a priori arbitrary, and additional physical consideration and empirical observations are re- quired to constrain it. The resulting action of the Lorentz group on $p$ becomes nonlinear

$$
p \rightarrow \mathcal{U}_{M_{P}}\left(L \cdot \mathcal{U}_{M_{P}}^{-1}(p)\right),
$$

where $L \in S O(3,1)$. The Casimir associated to these deformed symmetries has the general form of Eq. (1).

The nonlinear relation between $p$ and $\pi$ can always be interpreted as a coordinate change, so a priori it has no physical consequence. To encode new physics, additional physical inputs are required, and there are many ways to do so. For instance, the action of the Lorentz symmetry on space-time can be specified and, combined with its nonlinear action on $p$, it will generally imply a nontrivial addition for the coordinate $p$ for multiparticle states. Noncommutative geometry $[28,29]$ is an implementation of that approach. Alternatively, the coordinates $p$ and $\pi$ can be given a direct physical interpretation in terms of other fields. In Refs. [8-10], it was suggested that $p$ is the momentum as measured with respect to a physical RF while $\pi$ is the kinematical momentum. ${ }^{1}$ More explicitly, consider a space-time reference frame, that is a tetrad $e^{\mu}{ }_{\alpha}$, where $\mu$ labels space-time coordinates and $\alpha$ labels different vectors, independent of the chart. If a particle has a momentum $\pi_{\mu}$, the measured components $p_{\alpha}$ of this momentum are defined as

$$
p_{\alpha}=\pi_{\mu} e_{\alpha}^{\mu} .
$$

In Minkowski space-time, the tetrad is trivial $e^{\mu}{ }_{\alpha} \sim \delta^{\mu}{ }_{\alpha}$ so $\pi$ and $p$ coincide. Upon change of reference frame ${ }^{2}$

$$
e^{\mu}{ }_{\alpha} \rightarrow \bar{e}_{\alpha}^{\mu}=L_{\alpha}^{\beta} e_{\beta}^{\mu},
$$

the measured components of the momentum are transformed as

$$
p_{\alpha}^{\prime}=\pi_{\mu} \bar{e}_{\alpha}^{\mu}=L_{\alpha}^{\beta} e_{\beta}^{\mu} \pi_{\mu}=L_{\alpha}^{\beta} p_{\beta} .
$$

Thus, $p$ carries a linear realization of the Lorentz symmetries. Notice how the Lorentz transformation produces linear combinations among the different vectors labeled by $\alpha$. It is not affecting the space-time indices which are chart dependent and therefore not physical.

To obtain a nonlinear realization of the symmetry, we consider a quantum theory and assume that there is an interaction between the RF and the system of interest, leading to a nontrivial "mixing" between them. Thus, $\pi$, $e_{\alpha}$, and $p_{\alpha}$ will henceforth denote quantum operators. Without a complete theory of QG, it is not possible to fully specify what this interaction should be. Nonetheless, it has been argued that an effective treatment of the quantumgravitational fluctuations of the tetrad field can produce such mixing. Aloisio et al. [10] modeled a particle travel-

\footnotetext{
${ }^{1}$ In this setting, the space-time construction is not completely clear, and the authors did not discuss multiparticles states.

${ }^{2}$ We consider only Lorentz transformation and leave out translations for the sake of clarity.
} 
ing in a fluctuating space-time by adding a stochastic term to the tetrad field. The measured momentum, they argued, should then be defined with respect to an average tetrad

$$
E_{\alpha}^{\mu}\left(e, \pi, M_{P}\right)=\left\langle e_{\alpha}^{\mu}\right\rangle,
$$

where $\langle\cdot\rangle$ denotes the average of a quantity over a spacetime region of size dictated by the de Broglie wavelength of the particle $\lambda=1 / E$.

Making further assumptions about the behavior of the fluctuations, they argued that this average can be encoded in the form of a reversible nonlinear map

$$
e_{\alpha}^{\mu} \stackrel{U_{M_{P}}}{\rightarrow} E_{\alpha}^{\mu}\left(e, \pi, M_{P}\right)
$$

Lorentz transformations act linearly on the tetrad field $e_{\alpha}$, so their effect on the average tetrad $E_{\alpha}$ is specified by the following commutative diagram:

$$
\begin{array}{ccc}
e^{\mu}{ }_{\alpha} & \rightarrow^{L} & \bar{e}^{\mu}{ }_{\alpha}=L^{\beta}{ }_{\alpha} e^{\mu}{ }_{\beta} \\
\downarrow \mathcal{U}_{M_{P}} & & \downarrow \mathcal{U}_{M_{P}} \\
E^{\mu}{ }_{\alpha} & \rightarrow{ }^{\tilde{L}} & \bar{E}^{\mu}{ }_{\alpha} .
\end{array}
$$

This, in turn, induces a nonlinear transformation of the measured momentum $p$ :

$p_{\alpha}=\pi_{\mu} E^{\mu}{ }_{\alpha} \rightarrow p_{\alpha}^{\prime}=\pi_{\mu} \bar{E}_{\alpha}^{\mu}=\pi_{\mu} \mathcal{U}_{M_{P}}\left(L \cdot \mathcal{U}_{M_{P}}^{-1}(E)\right)$.

When the fluctuations have appropriate symmetries, the map $\mathcal{U}_{M_{P}}$ takes the simple form $E^{\mu}{ }_{\alpha}=F\left(e, \pi, M_{P}\right) e^{\mu}{ }_{\alpha}$, and $F\left(e, \pi, M_{P}\right) \rightarrow 1$ when $M_{P} \rightarrow \infty$. For example, $p_{\alpha}=$ $F\left(e, \pi, M_{P}\right) e_{\alpha}^{\mu} \pi_{\mu}$ with

$$
F\left(e, \pi, M_{P}\right)=\frac{1}{\sqrt{1-\frac{\pi_{\mu} e_{0}^{\mu}}{M_{P}}}}
$$

would generate the analog of the Magueijo-Smolin dispersion relation [30].

In short, the physical picture proposed in Refs. [8-10] is that physical observables are defined with respect to RF, and any interaction between the system of interest and the $\mathrm{RF}$ (including interactions required to perform a measurement) will modify the state of the RF, and hence the value of measured quantities. This picture is quite elegant and largely agrees with the conclusions reached in Ref. [27] in the context of quantum information. Ironically however, the mechanism outlined above does not exactly fit into this picture. The mixing between the system and the RF is caused not by an interaction or measurement, but indirectly by setting a coarse-grained scale that depends on the energy of the measured particle. The model we will consider in the next section adheres more closely to the original philosophy [8].

\section{DIRECTIONAL REFERENCE FRAME}

\section{A. Quantum gyroscopes}

The previous section presented an argument for the deformation of the Lorentz symmetry caused by a mixing between the system of interest and the RF. To render the analysis of this proposal more tractable, we will consider instead the effect of measurements on a directional RF, i.e. we shift the analysis from $S O(3,1)$ to $S U(2)$. The constraint then is that all physical observables must be rotationally invariant: if $J^{\mu}$ are the generators of rotation, only observables $O$ with $\left[O, J^{\mu}\right]=0$ for $\mu=1,2,3$ are physical. $^{3}$ Thus, our primary object of study is basically a gyroscope, a physical system that singles out a particular direction in three-dimensional space.

The quantum analog of a gyroscope is a system with a large amount of spin. The state of the gyroscope is described by a density matrix $\rho$ in the enveloping algebra of the spin- $\ell$ irreducible representation (irrep) of $s u(2)$. Throughout, we will consider $\ell$, or equivalently the Hilbert space dimension $d=2 \ell+1$, as an indication of the size of the reference. In practice, gyroscopes are composite systems, built from a large number of elementary particles. For instance, the magnetization of a ferromagnet, built up from its component electron spins, can serve as a good gyroscope. In that case, the size $\ell$ of the gyroscope would be proportional to the number of electrons inside the ferromagnetic sample. However, this aspect does not affect our analysis whatsoever, so we will henceforth refer to the gyroscope as if it was a single particle with a large spin.

A gyroscope singles out one direction in threedimensional space. To obtain a complete directional RF, we need at least two gyroscopes. To conform with the notation of the previous section, we denote the generators of the spin- $\ell$ irrep of $s u(2)$ associated to each of these spins by $e^{\mu}{ }_{\alpha}$ for $\alpha=1,2$ and $\mu=1,2,3$. As above, the index $\alpha$ labels the different gyroscopes (in this case two of them) and $\mu$ is an internal chart-dependent index. These operators satisfy the commutation relations

$$
\left[e_{\alpha}^{\mu}, e_{\beta}^{\nu}\right]= \begin{cases}0 & \text { if } \alpha \neq \beta \\ \frac{i}{\sqrt{\ell(\ell+1)}} \epsilon_{\eta}{ }^{\mu \nu} e^{\eta}{ }_{\alpha} & \text { if } \alpha=\beta,\end{cases}
$$

where $\epsilon_{\eta}{ }^{\mu \nu}$ is the totally antisymmetric tensor with $\epsilon_{1}{ }^{23}=$ 1. Note that the unusual factor of $1 / \sqrt{\ell(\ell+1)}$ comes in due to the normalization we have chosen. Since each gyroscope is a spin- $\ell$ particle, this normalization is

$$
e_{\alpha}^{\mu} e_{\mu \alpha}=1, \quad \text { for } \alpha=1,2 .
$$

The complete quantum triad $\left(e^{\mu}{ }_{1}, e^{\mu}{ }_{2}, e^{\mu}{ }_{3}\right)$ is obtained by defining $e^{\rho}{ }_{3}=\epsilon^{\rho}{ }_{\mu \nu} e^{\mu}{ }_{1} e^{\nu}{ }_{2}$. This triad provides a complete quantum RF for three-dimensional space.

\footnotetext{
${ }^{3}$ In Appendix A, we provide the full algebra of constrained (or Dirac) observables.
} 
The state of each gyroscope $\rho_{\alpha} \alpha=1,2$ is a nonnegative trace-one operator in the enveloping algebra of the spin- $\ell$ irrep generators $e^{\alpha}{ }_{\mu}$, i.e. a $d \times d$ matrix. The complete state of the RF is thus $\rho=\rho_{1} \otimes \rho_{2}$. Similarly to what was done in previous section, we can define the average triad:

$$
E_{\alpha}^{\mu}=\left\langle e^{\mu}{ }_{\alpha}\right\rangle=\operatorname{Tr}\left\{e^{\mu}{ }_{\alpha} \rho\right\} .
$$

Since it will be important later, we point out that this quantum average has a slightly different interpretation than the space-time average used in the previous section. This definition also implies that $\vec{E}_{3}=\vec{E}_{1} \times \vec{E}_{2}$. The spatial direction singled out by gyroscope $\alpha$ is thus parallel to $\vec{E}_{\alpha}=\left(E_{\alpha}^{1}, E_{\alpha}^{2}, E_{\alpha}^{3}\right)$. Note that for $\alpha=1,2$,

$$
0 \leq \vec{E}_{\alpha} \cdot \vec{E}_{\alpha} \leq 1-\frac{1}{\ell+1} .
$$

This bound is saturated when the gyroscopes are in socalled coherent states [31]. In fact, the equality $\vec{E}^{\alpha} \cdot \vec{E}^{\alpha}=$ $1-\frac{1}{\ell+1}$ with $E_{\alpha}$ given by Eq. (14) can be taken as the definition of a coherent state $\rho^{\alpha}$. Coherent states are in some sense most classical as they have the largest amount of spin concentrated in some direction.

\section{B. Quantum measurements}

Gyroscopes can be used to measure the spin of other "source" particles along the axis of rotation of the gyroscope. We will suppose that these are spin- $j$ particles with $j \ll \ell$. The $2 j+1$ dimensional irreducible representation of $s u(2)$ is generated by $\pi_{\mu}$. Once again, the subscript $\mu=$ $1,2,3$ is a chart-dependent internal index. The state of the source particles are $(2 j+1) \times(2 j+1)$ density matrix $\sigma$ in the enveloping algebra of the $\pi_{\mu}$.

Since only rotationally invariant observables have physical meaning, one can used the average triad $E^{\mu}{ }_{\alpha}$ introduced above to define the semiclassical relational coordinates of the source particles

$$
p_{\alpha}=\pi_{\mu} E^{\mu} .
$$

The semiclassical coordinates can be decomposed into a sum of $2 j+1$ orthogonal projectors $P_{\alpha}^{m}, m=-j, \ldots, j$ associated with distinct eigenvalues $\left|\vec{E}_{\alpha}\right| m$. For instance, the semiclassical coordinate associated to the average triad element say $\vec{E}_{\alpha}=(0,0,1)$ [which, as a consequence of Eq. (15), is only possible when $\ell \rightarrow \infty$ ] is simply $\pi_{3}$. A measurement of this semiclassical coordinate has the usual $m=-j, \ldots, j$ outcomes associated to the spin of the particle along the third axis.

One can easily verify the following commutation relation for the semiclassical coordinates

$$
\begin{gathered}
{\left[p_{1}, p_{2}\right]=i p_{3}} \\
{\left[p_{2}, p_{3}\right]=i p_{1} \vec{E}_{2} \cdot \vec{E}_{2}-i p_{2} \vec{E}_{1} \cdot \vec{E}_{2}}
\end{gathered}
$$

$$
\left[p_{3}, p_{1}\right]=i p_{2} \vec{E}_{1} \cdot \vec{E}_{1}-i p_{1} \vec{E}_{1} \cdot \vec{E}_{2} .
$$

These relations reduce to the usual $s u(2)$ relations when both gyroscopes are in coherent states rotating about perpendicular axes, and $\ell \rightarrow \infty$. (That the two gyroscopes be perpendicular has no fundamental significance because one can always take different linear combinations of the $\left.p_{\alpha}\right)$.

As suggested by the name, the semiclassical coordinates do not take into account the full quantum nature of the gyroscopes. This is because the triad $E^{\mu}{ }_{\alpha}$ has the physical meaning of an average, which only acquires an operational meaning in the presence of an ensemble. We thus define the quantum relational coordinates of the source particles as

$$
\mathfrak{p}_{\alpha}=\pi_{\mu} e^{\mu}{ }_{\alpha}
$$

These are physical observables as they commute with the generators of rotation. While all $\pi^{\mu}$ and $p_{\alpha}$ are operators on the Hilbert space of the source particle, the operators $\mathfrak{p}_{\alpha}$ act on the combined Hilbert space of the source particle and the gyroscope $\alpha$. As the different font indicates, the operator $\mathfrak{p}$ does not have a counterpart in the discussion of Sec. II. We recover the semiclassical coordinates by taking the average of the quantum coordinates over the state of the gyroscopes

$$
\operatorname{Tr}_{\mathrm{RF}}\left\{\mathfrak{p}_{\alpha} \rho\right\}=p_{\alpha},
$$

where $\operatorname{Tr}_{\mathrm{RF}}$ denotes the partial trace over the Hilbert space of the RF, and as above $\rho=\rho_{1} \otimes \rho_{2}$ is the state of the RF. Despite this relation, a measurement of $p_{\alpha}$ differs from a measurement of $\mathfrak{p}_{\alpha}$ in two fundamental ways:

(1) The measurement of $\mathfrak{p}_{\alpha}$ will only be an approximation of what would be obtained by using the corresponding classical reference, i.e. $p_{\alpha}$.

(2) Each time the reference is used to measure $\mathfrak{p}_{\alpha}$, it suffers an inevitable "back-action" which ultimately changes the character of future measurements.

We will describe these points in details, but they can be understood intuitively as follows. The first point is a consequence of the quantum fluctuations of the quantum gyroscope. This effect is minimized when the state of the gyroscope $\rho_{\alpha}$ is in a coherent state, which minimizes the fluctuations $\sum_{\mu}\left\langle e^{\mu \alpha}\right\rangle^{2}$.

The second point is a consequence of the fact that $\mathfrak{p}_{\alpha}$ does not commute with any of the $e^{\mu}{ }_{\alpha}$ :

$$
\left[e^{\mu}{ }_{\alpha}, \mathfrak{p}_{\alpha}\right]=\frac{i}{\ell} \epsilon_{\nu \eta}^{\mu} e_{\alpha}^{\nu} \pi^{\eta} .
$$

By the uncertainty principle, a measurement of $\mathfrak{p}_{\alpha}$ will thus alter the value of $e_{\alpha}$, and so disturb any future measurements that make use of that gyroscope.

The discrepancy between the semiclassical and quantum triads $p_{\alpha}$ and $\mathfrak{p}_{\alpha}$ also manifests itself in their commutation relations. The analog of Eqs. (17)-(19) for the quantum 
triad is

$$
\begin{aligned}
& {\left[\mathfrak{p}_{1}, \mathfrak{p}_{2}\right]=i \mathfrak{p}_{3}} \\
& {\left[\mathfrak{p}_{2}, \mathfrak{p}_{3}\right]=i \mathfrak{p}_{1}+-i e^{\mu}{ }_{2} e_{\mu 1} \mathfrak{p}_{2}+i \frac{\mathfrak{p}_{1}, \mathfrak{p}_{2}-\vec{\pi} \cdot \vec{\pi} e^{\mu}{ }_{2} e_{\mu 1}}{\sqrt{\ell(\ell+1)}}} \\
& {\left[\mathfrak{p}_{3}, \mathfrak{p}_{1}\right]=i \mathfrak{p}_{2}+-i \mathfrak{p}_{1} e^{\mu}{ }_{2} e_{\mu 1}+i \frac{\mathfrak{p}_{1}, \mathfrak{p}_{2}-\vec{\pi} \cdot \vec{\pi} e^{\mu}{ }_{2} e_{\mu 1}}{\sqrt{\ell(\ell+1)}}}
\end{aligned}
$$

which differs from Eqs. (18) and (19) by the addition of a third term, that vanishes in the limit $\ell \rightarrow \infty$.

\section{Approximate measurement}

To understand in what sense a measurement of $\mathfrak{p}_{\alpha}$ yields an approximation of a measurement of $p_{\alpha}$ requires basic elements of the theory of generalized measurement. Because of its special nature, the third component of the triad $\mathfrak{p}_{3}=-i\left[\mathfrak{p}_{1}, \mathfrak{p}_{2}\right]$ requires a separate analysis that does not provide additional insights into the problem of interest. For simplicity, we will henceforth concentrate on $\alpha=1,2$. The spectral theorem can be used to decompose each operator $\mathfrak{p}_{\alpha}$ as

$$
\mathfrak{p}_{\alpha}=\sum_{k=\ell-j}^{\ell+j} \lambda^{k} \Pi_{\alpha}^{k}
$$

where the eigenvalues

$$
\lambda^{k}=\frac{k(k+1)-\ell(\ell+1)-j(j+1)}{2 \sqrt{\ell(\ell+1)}}
$$

converge to the eigenvalues $k-\ell=-j, \ldots, j$ of $p_{\alpha}$ when $\ell \rightarrow \infty$. Like the quantum coordinates $\mathfrak{p}_{\alpha}$ themselves, the spectral projectors act jointly on the gyroscopes and the source particles. Their explicit form is

$$
\Pi_{\alpha}^{k}=\frac{1}{N^{k}} \prod_{k^{\prime} \neq k}\left(\lambda^{k^{\prime}}-\mathfrak{p}_{\alpha}\right),
$$

where the normalization factor is

$$
N^{k}=\prod_{k^{\prime} \neq k}\left(\lambda^{k^{\prime}}-\lambda^{k}\right) .
$$

These projectors are nonlinear functions of the relational coordinates $\mathfrak{p}_{\alpha}$; more precisely they are polynomials of degree $2 j$. When the gyroscopes are in state $\rho$ and the source particle is in state $\sigma$, a measurement of $\mathfrak{p}_{\alpha}$ will produce the outcomes $\lambda^{k}$ with probability

$$
\operatorname{Pr}\left(\lambda^{k}\right)=\operatorname{Tr}\left\{\Pi_{\alpha}^{k} \rho \otimes \sigma\right\}
$$

This equation can be expressed as

$$
\operatorname{Pr}\left(\lambda^{k}\right)=\operatorname{Tr}\left\{\Lambda_{\alpha}^{k} \sigma\right\}
$$

which only involves the state of the source particles $\sigma$, where the generalized measurement operators $\Lambda_{\alpha}^{k}$ are defined as

$$
\Lambda_{\alpha}^{k}=\operatorname{Tr}_{\mathrm{RF}}\left\{\Pi_{\alpha}^{k} \rho\right\} .
$$

Note that $\Lambda^{k}$ are not projectors in general, so Eq. (28) is a generalization of Born's probability rule to the case of positive operator valued measurement (POVM).

When both gyroscopes are in coherent states and $\ell \rightarrow$ $\infty$, the POVM elements $\Lambda_{\lambda}^{k}$ are equal to the spectral projectors of the semiclassical observables $P_{\alpha}^{k}$ defined above. In that limit, measurements of $p_{\alpha}$ and $\mathfrak{p}_{\alpha}$ coincide. In general however, the POVM elements $\Lambda_{\alpha}^{k}$ are "coarsegrained" versions of $P_{\alpha}^{k}$, mixing the different $k$ components:

$$
\Lambda_{\alpha}^{k}=\left(1-\epsilon^{k}\right) P_{\alpha}^{k}+\sum_{k^{\prime} \neq k} \epsilon^{k^{\prime}, k} P_{\alpha}^{k^{\prime}},
$$

where all the "mixing terms" $\epsilon$ are of order $1 / \ell$. Thus, any finite quantum RF yields an approximate measurement of the associated semiclassical observable [32,33]. When the gyroscopes are not in coherent states, the mixing terms become more important. They are of order $1-\left|\vec{E}_{\alpha}\right|$, cf. Eq. (15).

\section{Back-action}

The second aspect that distinguishes quantum relational coordinates from semiclassical ones is the back-action experienced by the RF. This effect is best described using the generalized theory of quantum dynamics. When a measurement of $\mathfrak{p}_{\alpha}$ is performed and outcome $\lambda^{k}$ is obtained, the joint state the source particle and RF is

$$
\frac{\Pi_{\alpha}^{k}(\rho \otimes \sigma) \Pi_{\alpha}^{k}}{\operatorname{Pr}\left(\lambda^{k}\right)}
$$

according to von Neumann's measurement postulate. Averaging over the measurement outcomes and tracing the state of the source particle gives the average state of the RF after the measurement is performed:

$$
\rho^{\prime}=\sum_{k} \operatorname{Tr}_{S}\left\{\Pi_{\alpha}^{k}(\rho \otimes \sigma) \Pi_{\alpha}^{k}\right\}
$$

where $\operatorname{Tr}_{S}$ denotes the partial trace over the Hilbert space of the source particle. Decomposing the state of the source particle in terms of its eigenstates $\sigma=\sum_{i} s_{i}\left|\phi_{i}\right\rangle\left\langle\phi_{i}\right|$, and performing the partial trace in that same basis gives

$$
\rho^{\prime}=\sum_{k, i, i^{\prime}} s_{i}\left\langle\phi_{i^{\prime}}\left|\Pi_{\alpha}^{k}\right| \phi_{i}\right\rangle \rho\left\langle\phi_{i}\left|\Pi_{\alpha}^{k}\right| \phi_{i^{\prime}}\right\rangle .
$$

Thus, the back-action on the RF is described by a dynamical map, which can be written in a Kraus form [34]

$$
\rho^{\prime}=\mathcal{E}_{\alpha}(\rho)=\sum_{a} K_{a} \rho K_{a}^{\dagger}
$$

where for $a=\left(k, i, i^{\prime}\right)$ the Kraus operators $K_{a}=$ $\sqrt{s_{j}}\left\langle\phi_{i^{\prime}}\left|\Pi_{\alpha}^{k}\right| \phi_{i}\right\rangle$ are operators on the Hilbert space of the $\mathrm{RF}$, and satisfy $\sum_{a} K_{a}^{\dagger} K_{a}=1$. The subscript $\alpha$ on $\mathcal{E}$ reflects the fact that the measurement of different $\mathfrak{p}_{\alpha}$ will induce 
different back-actions on the RF. Expressed in the Heisenberg picture, this map modifies the quantum triad according to

$$
\mathcal{E}_{\alpha}^{\dagger}\left(e^{\mu}{ }_{\beta}\right)=\sum_{a} K_{a}^{\dagger} e^{\mu}{ }_{\beta} K_{a} .
$$

By definition of the spectral projectors $\Pi_{\alpha}^{k}$ Eq. (31), the map $\mathcal{E}_{\alpha}$ is a nonlinear function of the relational coordinates $\mathfrak{p}_{\alpha}$.

Thus we arrive at the conclusion: The measurement of the relational quantum coordinate of a source particle induces a back-action on the RF, which in general is a nonlinear function $\mathcal{E}_{\alpha}$ of the quantum relational coordinate $\mathfrak{p}_{\alpha}$. This observation supports the proposal of Refs. [8-10], but important distinctions will be discussed in Sec. V. In what follows, we examine the possibility of detecting this effect experimentally.

\section{DETECTING THE DEFORMATION}

A constrained measurement of the spin of a source particle induced a nonlinear back-action on the RF. This will have the effect of changing the measurement outcomes of subsequent measurements that make use of that RF. Since the effect is expected to be tiny, this modification of measurement outcome probabilities can only be detected by repeating the experiment several times and accumulating statistics. Thus, to understand the nature of any experiment aimed at detecting this effect, we must first understand the effect of sequential measurements on the RF.

\section{A. Consecutive relational measurements}

In this section, we describe the dynamics incurred by the $\mathrm{RF}$ when it is used to sequentially measure the coordinates of particles drawn from a fixed ensemble. All source particles are assumed to be in the same state $\sigma$. Each time the gyroscope $\alpha$ is used to measure the coordinate of a source particle, it experiences a back-action described by the quantum map $\mathcal{E}_{\alpha}$. After $t$ such measurements, the state of the gyroscope is given by $\mathcal{E}_{\alpha} \circ \mathcal{E}_{\alpha} \circ \cdots \circ$ $\mathcal{E}_{\alpha}\left(\rho_{\alpha}\right)=\mathcal{E}_{\alpha}^{t}\left(\rho_{\alpha}\right)$, where $\circ$ denotes the usual composition.

The effect of this map was studied in great details for the case $j=\frac{1}{2}$ in [27]. In that case, the kinematics is given by the spin- $\frac{1}{2}$ generators of $s u(2)$, i.e. the Pauli matrices

$$
\begin{gathered}
\pi^{1}=\frac{1}{2}\left(\begin{array}{ll}
0 & 1 \\
1 & 0
\end{array}\right), \quad \pi^{2}=\frac{1}{2}\left(\begin{array}{cc}
0 & i \\
-i & 0
\end{array}\right), \\
\pi^{3}=\frac{1}{2}\left(\begin{array}{cc}
1 & 0 \\
0 & -1
\end{array}\right) .
\end{gathered}
$$

When the gyroscopes' state $\rho_{\alpha}$ are coherent states and $\ell \gg$ 1 , the results can be summarized as follows. For $\alpha=1,2$, the leading order of $\mathcal{E}_{\alpha}$ in $1 / \ell$ is

$$
\begin{aligned}
\mathcal{E}_{\alpha}\left(\rho_{\alpha}\right) & \approx \rho_{\alpha}-\frac{i}{\ell} \epsilon_{\mu \nu \eta} E^{\mu}{ }_{\alpha}\left\langle\pi^{\nu}\right\rangle \sin \theta\left[e^{\eta}{ }_{\alpha}, \rho_{\alpha}\right] \\
& =\mathcal{U}_{\alpha}^{\dagger}(e, \pi, 1 / \ell)\left(\rho_{\alpha}\right)
\end{aligned}
$$

where $\cos \theta=\vec{E}_{\alpha} \cdot\langle\vec{\pi}\rangle /|\langle\vec{\pi}\rangle|$. In other words, the RF undergoes a rotation by an angle $\sin \theta|\langle\vec{\pi}\rangle| / \ell$ about the axis $\vec{E}_{\alpha} \times\langle\vec{\pi}\rangle$, where $\langle\vec{\pi}\rangle=\operatorname{Tr}\{\vec{\pi} \sigma\}$ is the average spin of the source particle. $U$ is a unitary rotation and hence reversible transformation of the RF.

Since each application of the map $\mathcal{E}_{\alpha}$ rotates the gyroscope towards the axis of polarization of the source particles $\langle\vec{\pi}\rangle$, the gyroscope will eventually line up perfectly with the source particles. This happens in a time proportional to $\ell$. In the Heisenberg picture, the cumulative effect of this reversible component of the back-action after $t$ measurements is

$$
e_{\alpha}^{\mu} \stackrel{t}{\rightarrow} \mathcal{U}_{\alpha}^{t}(e, \pi, 1 / \ell)\left(e_{\alpha}^{\mu}\right) .
$$

We note that rotations map coherent states to coherent states, so in leading order the RF remains in a coherent state.

The transformation $\mathcal{E}_{\alpha}$ is not strictly unitary however, and nonunitary effects become manifest at higher orders. To understand this effect, it is helpful to think of the source particle as an "environment" that couples to the RF. It is well understood that coupling to an environment typically implies a noisy nonunitary evolution of the RF [35]. This is a consequence of the fact that after the measurement is performed, the source particle and the RF are in general entangled with one another.

Thus, the state of each gyroscope will typically deteriorate due to the nonunitary effects of $\mathcal{E}_{\alpha}$. As explained in Sec. III C, the measurement of the quantum relational coordinate $\mathfrak{p}_{\alpha}$ is in general an approximation of the semiclassical relational coordinate $p_{\alpha}$. The quality of the approximation depends on the state of the gyroscope-more precisely it depends on the norm of the average polarization $\vec{E}_{\alpha}$. When a gyroscope is initially in a coherent state, this norm is maximal. As a consequence of the measurements of the source particles, $\left|\vec{E}_{\alpha}\right|$ will in general decrease, and thus the approximate equivalence between $p_{\alpha}$ and $\mathfrak{p}_{\alpha}$ will deteriorate. This happens after a number of measurements proportional to $\ell$. At a later stage, when the unitary rotation has brought the RF in a state parallel to the source particles, $\left|\vec{E}_{\alpha}\right|$ will increase to become near maximal again. Figure 3 of Ref. [27] summarizes this dynamics.

Since the details of the dynamics are not important in the following analysis, we will assume that the essential features derived in Ref. [27] that we have just summarized extend beyond the case $j=\frac{1}{2}$. This hypothesis is corroborated by the fact that it is always possible to imagine a particle with spin $j>\frac{1}{2}$ as composed of $2 j$ spin- $\frac{1}{2}$ particles in a symmetric state. Thus, the map induced on the RF by a spin- $j$ particle relates to the map obtained from $2 j$ appli- 
cations of the map induced by a spin- $\frac{1}{2}$ particle. Recent work [36] on the case $j>\frac{1}{2}$ also supports this picture.

\section{B. Possible experimental setting}

Now that we have described the dynamics incurred by the RF when used to consecutively measure the spin of source particles, we are in a position to determine whether this effect is detectable in principle. We do this by analyzing gendanken experimental settings where signatures of such deformation could be detected.

The simplest experimental verification of the symmetry transformations of a RF uses spin- $\frac{1}{2}$ source particles and proceeds as follows:

(1) Use the RF to measure the quantum relational coordinate of $t$ source particles.

(2) Tabulate the statistics of the $\lambda_{\alpha}^{ \pm}$outcomes and compute $\operatorname{Pr}_{\alpha}\left(\lambda^{ \pm}\right)$.

(3) Apply a rotation to the RF from its original position by an angle $\theta, e^{\mu}{ }_{\alpha} \rightarrow \Lambda^{\alpha}{ }_{\beta} e^{\mu}{ }_{\alpha}$, and repeat steps 1 and 2 for a different set of $t$ source particles, drawn from the same ensemble.

According to Mallus' law, the probability of outcome of say $\lambda^{+}$as a function of the angle $\theta$ should follow a cosine

$$
\operatorname{Pr}_{\alpha}\left(\lambda^{+}, \theta\right)=\frac{1+Q \cos \left(\theta+\varphi_{\alpha}\right)}{2},
$$

where $\varphi_{\alpha}$ are some fixed offsets and the visibility $Q$ is between 0 and 1 .

To verify this prediction within accuracy $\epsilon \ll 1$, one needs to perform each set of measurements on $t \approx 1 / \epsilon^{2}$ source particles. ${ }^{4}$ These measurements will induce a backaction on the RF. If $t$ is too large, this back-action will completely deteriorate the RF, i.e. it will result in a visibility $Q=0$. Since this effect takes place after a number of measurements $\propto \ell$ [27], it places a constraint on the size of the gyroscopes $\ell \gg 1 / \epsilon^{2}$. In that regime, the dominant effect of the back-action will be to rotate the RF by an angle $\Delta \theta \approx t / \ell=1 / \ell \epsilon^{2}$, cf. Eqs. (36) and (37).

Because of this additional rotation, the probability of the measurement outcomes in the subsequent setting will be altered. In the second round of measurements for instance, the measurement outcome probability is given by Eq. (39), but with $\theta$ replaced by $\theta+\Delta \theta$ on the right-hand side, where $\Delta \theta$ is the back-action rotation caused by the first set of measurements. This effect is only significant if the resulting probability differs from the predicted probability by an amount greater than the measurement accuracy $\epsilon$. To leading order, the correction to the probability is simply

\footnotetext{
${ }^{4}$ The possibility of preparing the source particles in a massively entangled state could improve this bound to $t \approx 1 / \epsilon$ [37]. However, we assume here that the source particles are not controlled by the experimentalist, for instance they could have an astrophysical origin. In that case, it can always be assumed that they are all in the same (possibly unknown) state $\sigma$ [38].
}

proportional to $\Delta \theta$, so we obtain the constraint $\Delta \theta \approx$ $1 / \ell \epsilon^{2}>\epsilon$.

Combining this with the constraint established in the previous paragraph, we conclude that the measurement back-action causes a rotation of the RF that is perceptible with accuracy $\epsilon \ll 1$ only if

$$
\frac{1}{\epsilon^{2}} \ll \ell<\frac{1}{\epsilon^{3}} .
$$

This places important constraints on the regime where these effects are relevant, but demonstrate the possibility of detecting them in principle.

\section{DISCUSSION}

In this section, we discuss the connections and discrepancies between the directional RF model and deformed special relativity.

\section{A. Deformation}

When a quantum directional RF is used to perform a constrained measurement on source particles, we have shown that

(1) The RF undergoes a transformation $e^{\mu}{ }_{\beta} \rightarrow$ $\mathcal{E}_{\alpha}^{\dagger}\left(e^{\mu}{ }_{\beta}\right)$, where $\mathcal{E}_{\alpha}$ is in general a nonlinear function of the quantum relational coordinated $\mathfrak{p}_{\alpha}$.

(2) The map $\mathcal{E}_{\alpha}$ has two components: a unitary $\mathcal{U}_{\alpha}$ and hence reversible rotation, and a noisy irreversible component caused by the act of measurement that creates correlations between the RF and the source particles. The reversible effect is dominant when the $\mathrm{RF}$ is in a coherent state and its size $\ell \gg t$, where $t$ is the number of measurements.

(3) The model we have analyzed admits a regime where these effects can be detected experimentally.

The first point supports the idea that the quantum fluctuations incurred by RF can lead to an effective deformation of the symmetries of the relational observables. As opposed to the models investigated in [8-10] however, this deformation is truly a dynamical effect. Indeed, to measure the relational coordinate $\mathfrak{p}_{\alpha}$ requires somehow coupling the RF and the source particles. For instance, it can be achieved by turning on a Heisenberg interaction between the two systems and measuring their total energy, which would be directly proportional to the eigenvalues $\lambda_{\alpha}^{k}$ of $\mathfrak{p}_{\alpha}$. Note, moreover, that it is really the coupling that causes the back-action, and the same dynamical map $\mathcal{E}_{\alpha}$ would be obtained regardless of whether the total energy was actually measured. Thus, our conclusions are relatively insensitive to the details of the mixing between the RF and the source particles.

The second point is also crucial to the proposal of [810]: only when the back-action has an inverse can it be interpreted as a symmetry deformation, cf. Eqs. (9) and (10), otherwise it is better described simply as noise. The 
question of whether a map is reversible or not on a subsystem has been studied in detail in quantum information science, in the context of quantum error correction, see [39] for the latest developments. The question of approximate reversibility - relevant to the present study - has also been studied in the context of quantum error correction [40].

While the leading effect of $\mathcal{E}_{\alpha}$ on the RF was found to be reversible in our model, it also presented an important irreversible component. In fact, depending on the details of the experiment - the number of times the RF is used, the polarization of the source particles, the size and initial state of the RF, etc.- this irreversible component can become the dominant effect of the back-action [25]. In that case, the mixing between the RF and source particles would not yield a symmetry deformation; rather, it would reveal itself as a fundamental source of noise.

The existence of irreversible processes in an effective low-energy theory can be understood quite simply on general grounds. The effective theory is meant to describe the dynamics of the low-energy degrees of freedom once the high-energy degrees of freedoms have been traced out. In the presence of correlations between the low- and highenergy sectors-which are to some extent inevitable in interacting theories - the resulting dynamics will be nonunitary, with the high-energy sector acting as an environment decohering the low-energy sector. The approximate reversibility of the effective theory is a manifestation of the "decoupling principle" according to which the dominant effect of the high-energy theory on the low-energy sector can be taken into account by renormalizing its parameters. Thus, according to this principle, the low-energy sector is well approximated by a Hamiltonian, and hence reversible, dynamics.

The third point, regarding the measurability of the effect, is difficult to address in a more general context. This is partly due to the imperfect analogy between the model studied here and the situation faced by DSR. For instance, $S U(2)$ is compact while $S O(3,1)$ is not, DSR imposes a natural minimum mass $M_{P}$ while the small parameter $1 / \ell$ in our model was tunable. These aspects will most likely alter the quantitative aspect of our analysis, and impact the analysis of Sec. IV B.

Despite the common features shared between the gyroscope model and DSR, there exists an important caveat in the analogy. As mentioned above, the back-action on the $\mathrm{RF}$ is really a dynamical effect: the state of the RF after it has coupled to the source particle differs from its state prior to this interaction. The counterpart of Eq. (8) in the gyroscope model is thus

$$
e_{\alpha}^{\mu}(t) \stackrel{U_{\beta}}{\rightarrow} e^{\mu}{ }_{\alpha}\left(t^{\prime}\right),
$$

where $t$ is the time immediately before the measurement and $t^{\prime}$ is the time immediately after, and $\mathcal{U}_{\alpha}$ is the reversible approximation to $\mathcal{E}_{\alpha}$. Contrary to the proposal of [8], this does not directly imply a nonlinear relation between the kinematical coordinate $\pi$ and its measured components $\mathfrak{p}_{\alpha}$.

However, the value of $\mathfrak{p}_{\alpha}$, like all quantum observables, can only be known probabilistically. Thus, as argued in Sec. IV B, a high precision measurement of the relational coordinate requires repeating over a large ensemble of identically prepared source particles. Since each one of these measurements induces a nonlinear back-action on the $\mathrm{RF}$, the ensemble average $\left\langle\mathfrak{p}_{\alpha}\right\rangle_{\mathrm{ens}}$ will be nonlinearly related to the kinematical coordinate. Explicitly, this ensemble average is

$$
\begin{aligned}
\left\langle\mathfrak{p}_{\alpha}\right\rangle_{\mathrm{ens}} & =\frac{1}{t} \sum_{i=t}^{N} \operatorname{Tr}\left\{\mathcal{U}_{\alpha}^{t-1}\left(e^{\mu}{ }_{\alpha}\right) \pi_{\mu} \rho \otimes \sigma\right\} \\
& =\operatorname{Tr}\left\{\left\langle E^{\mu}{ }_{\alpha}\right\rangle_{\mathrm{ens}} \pi_{\mu} \sigma\right\},
\end{aligned}
$$

where we have defined $\left\langle E^{\mu}{ }_{\alpha}\right\rangle_{\text {ens }}$ as the time average of the kinematical coordinates of the RF $\left\langle E^{\mu}{ }_{\alpha}\right\rangle_{\mathrm{ens}}=$ $\frac{1}{t} \sum_{t} \operatorname{Tr}\left\{\mathcal{U}_{\alpha}^{t-1}\left(e^{\mu}{ }_{\alpha}\right) \rho\right\}$. From an operational standpoint, we thus recover an analogue of Eq. (8):

$$
e_{\alpha}^{\mu} \stackrel{\left\langle U_{\beta}\right\rangle_{\text {ens }}}{\rightarrow}\left\langle E^{\mu}{ }_{\alpha}\right\rangle_{\mathrm{ens}}
$$

only now the quantum average $\langle\cdot\rangle=\operatorname{Tr}\{\cdot \rho\}$ used in the definition of $E^{\mu}{ }_{\alpha}$, cf. Eq. (14), needs to be supplemented by a dynamical average as induced by the back-action. While this is an important conceptual difference, we note that the quantum average $\langle\cdot\rangle$ can only be estimated experimentally by repeating the measurement over a large ensemble, and so the dynamical average is inevitable.

\section{B. Multiparticle observables}

The deformation of the Lorentz symmetry implies a nontrivial construction of multiparticles observables. This is also the case in the gyroscope model, and the goal of this section is to briefly outline this analogy.

Different choices of deformation maps $\mathcal{U}_{M_{P}}$ will lead to different momenta addition rules, which can be either noncommutative (and possibly also nonassociative) or commutative (but still nontrivial). A typical example of the first class is the (associative) momenta addition arising in the bicrossproduct basis [28]:

$$
p_{0}^{(\mathrm{tot})}=p_{0}^{(1)}+p_{0}^{(2)}, \quad p_{i}^{(\mathrm{tot})}=e^{-p_{0}^{(2)} / M_{P}} p_{i}^{(1)}+p_{i}^{(2)} .
$$

Typically, noncommutative additions suffer from the "soccer ball problem": just like the single-particle momentum $p_{\alpha}^{(i)}$, the total momentum of two particles $p_{\alpha}^{(\mathrm{tot})}$ is also bounded by the Planck mass. This is clearly in conflict with everyday observations where the total momentum of an object composed of a large number of particle, e.g. a soccer ball, can be much greater than $M_{P}$. 
The commutative addition on the other hand can avoid this problem. A typical example of this class is obtained from the modified reference frame approach presented in Sec. II. The intrinsic momentum of a 2-particles system is $\pi^{(\mathrm{tot})}=\pi^{(1)}+\pi^{(2)}$. In Minkowski space-time, the measured total momentum is simply

$$
p_{\alpha}^{(\mathrm{tot})}=\left(\pi^{(1)}+\pi^{(2)}\right)_{\mu} e_{\alpha}^{\mu}=p_{\alpha}^{(1)}+p_{\alpha}^{(2)} .
$$

In the presence of a nonlinear relation between the kinematical tetrad and its average, we obtain instead

$$
p_{\alpha}^{(\mathrm{tot})}=\left(\pi^{(1)}+\pi^{(2)}\right)_{\mu} E^{\mu}{ }_{\alpha}\left(e,\left(\pi^{(1)}+\pi^{(2)}\right), \gamma M_{P}\right),
$$

where the rescaling factor $\gamma>1$ (related to the number of particles) is introduced to avoid the soccer ball problem $[30,41]$.

In the gyroscope model, the addition of vectors is straightforward: the total spin of two particles is

$$
\vec{\pi}^{(\mathrm{tot})}=\vec{\pi}^{(1)}+\vec{\pi}^{(2)}
$$

and so

$$
\left[\pi_{\mu}^{(1)}, \pi_{\nu}^{(2)}\right]=0, \quad\left[\pi_{\mu}^{(\mathrm{tot})}, \vec{\pi}_{\mu}^{(k)}\right]=0, \quad \text { with } k=1,2
$$

The total relational coordinates is defined as before:

$$
\mathfrak{p}_{\alpha}^{(\text {tot })}=\pi_{\mu}^{(\text {tot) }} e_{\alpha}^{\mu} \text {. }
$$

The quantum nature of the RF implies unusual features for these relational coordinates. For instance, the coordinates associated to distinct particles do not commute, e.g. for $\alpha=1,2$,

$$
\left[\mathfrak{p}_{\alpha}^{(1)}, \mathfrak{p}_{\alpha}^{(2)}\right]=\frac{\epsilon_{\rho}{ }^{\mu \nu}}{\sqrt{\ell(\ell+1)}} \pi_{\mu}^{(1)} \pi_{\nu}^{(2)} e_{\alpha}{ }^{\rho}
$$

which vanishes when $\ell \rightarrow \infty$. This noncommutativity is in fact related to the measurement back-action: by the uncertainty principle, measuring the relational coordinate of the first particle will alter the value of the relational coordinate of the second particle. Similarly, the physical spin of each particles does not commute with their total spin $\left[\mathfrak{p}_{\alpha}^{(k)}, \mathfrak{p}_{\alpha}^{(\mathrm{tot})}\right] \neq 0$ for $k=1,2$. Again following the uncertainty principle, measuring the total spin of two particles differs from measuring their individual spins and adding the outcomes.

While the gyroscope model is not plagued with the soccer ball problem, a similar problem arises when the $\mathrm{RF}$ is small compared to the size or number of measured particles. When $j>\ell$ for instance, the spectrum $\lambda^{k}$ of the quantum relational coordinate $\mathfrak{p}_{\alpha}$, cf. Eq. (24), is very different from the spectrum of the semiclassical coordinate $p_{\alpha}$. In fact, $p_{\alpha}$ always has $2 j+1$ distinct eigenvalues while $\mathfrak{p}_{\alpha}$ has at most $2 \ell+1$ of them. Relatedly, the spacing between the eigenvalues of $\mathfrak{p}_{\alpha}$ is $\lambda^{k+1}-\lambda^{k} \simeq 1+O\left(\frac{j}{\ell}\right)$ as opposed to 1 for the semiclassical coordinate, and this is noticeable when $j>\ell$.

Similarly, the total spin $\sum_{i} \mathfrak{p}_{\alpha}^{(i)}$ of an arbitrary large collection of spin- $\frac{1}{2}$ particles relative to a fixed gyroscope can only take on a maximum number $n$ of distinct values. This contrasts with the kinematical spin $\pi_{\mu}$ or semiclassical relational spin $p_{\alpha}$ that both have a range proportional to the number of particles. However, this "soccer ball problem" can be avoided by using a larger RF.

\section{CONCLUSION}

In light of recent analysis conducted in the context of quantum information science, we have examined the proposal that the back-action incurred by a quantum reference frame when used to measure a relational observable can lead to an effective deformation of its symmetries. This proposal was first put forth in a quantum-gravitational setting [8-10], to support the idea that quantum fluctuations of the gravitational field can be described at an effective level by deformed special relativity. The existence of an auxiliary (kinematical) momentum variable carrying a linear realization of the Lorentz group and related nonlinearly to the physical (relational) momentum is central to this proposal.

Our analysis led us to the conclusion that various forms of mixing between the RF and source particles-and, in particular, mixing caused by the measurement of a relational observable-will generally lead to a back-action on the RF that depends nonlinearly on the relational coordinates. Since the back-action alters the state of the RF only after the measurement, it does not directly imply a nonlinear relation between the kinematical and relational coordinates. However, a nonlinear relation arises on average, when the RF is used to sequentially measure many source particles. Since repeated measurements are necessary to reach the accuracy required to probe these tiny effects, we obtain an effective nonlinear relation between the kinematical and relational coordinates. This provides a concrete mechanism for the key component of the proposal [810]. If this latter is taken seriously as encoding DSR, our model shows that there is a priori no reason to restrict the symmetry deformation to the boost sector; the rotations could also be deformed. A DSR theory incorporating this feature should be considered and could provide new ways to experimentally constrain the deformation induced by quantum-gravitational effects. We leave this question for future investigation.

Other analogies between our simple model and DSR were also briefly discussed. In particular, multiparticle observables share many similar nontrivial properties, like noncommutativity and spectrum saturation (also known as the soccer ball problem in DSR). While these analogies are rather superficial, they may hint on a deeper connection worthy of further investigation. 
Finally, the important and unavoidable role of irreversibility in the measurement back-action was also discussed. When used to perform sequential measurements on distinct particles, the RF will generally degrade. This places important constraints on the measurability of the effective symmetry deformation, which is caused by the reversible component of the back-action. In our simple model, there exist a regime in which the back-action is dominated by a its reversible component, but it is not clear how this generalizes to a broader context.

\section{ACKNOWLEDGMENTS}

We thank Gerard Milburn, Kenny Pregnell, and Jon Yard for stimulating discussions that motivated parts of this work. D. P. is supported in part by the Gordon and Betty Moore Foundation through Caltech's Center for the Physics of Information, by the National Science Foundation under Grant No. PHY-0456720, and by the Natural Sciences and Engineering Research Council of Canada. F. G. would like to thank M. Nielsen for his kind hospitality at the University of Queensland where this project was initiated.

\section{APPENDIX: COMPLETE SET OF CONSTRAINED OBSERVABLES}

In this Appendix, we construct the complete algebra of constrained observables for a collection of spin particles constrained to be invariant under a global $S U(2)$ symmetry. We recover, in particular, the relational observables of Sec. III.

This algebra of constrained observables can be constructed using the Schwinger-Jordan representation which encodes the spin of a particle in a pair of constrained harmonic oscillators. Consider two quantum harmonic oscillators:

$$
\left[a, a^{\dagger}\right]=\left[b, b^{\dagger}\right]=1, \quad[a, b]=0 .
$$

These can be used to define spin operators

$$
\begin{aligned}
& J_{z}=\frac{1}{2}\left(a^{\dagger} a-b^{\dagger} b\right), \quad J_{+}=a^{\dagger} b, \\
& J_{-}=J_{+}^{\dagger}=a b^{\dagger}, \quad E=\frac{1}{2}\left(a^{\dagger} a+b^{\dagger} b\right) .
\end{aligned}
$$

The operators $J$ 's define a $s u(2)$ algebra while the total energy $E$ commutes with the $J$ 's and is thus a Casimir operator for $s u(2)$ :

$$
[E, \cdot]=0, \quad\left[J_{z}, J_{ \pm}\right]= \pm J_{ \pm}, \quad\left[J_{+}, J_{-}\right]=2 J_{z} .
$$

The spin representations at fixed $j$ 's are given by fixing the total energy $E$. Then diagonalizing the two operators $E$ and $J_{z}$, we obtain the simple correspondence between the $S U$ (2) usual basis $|j m\rangle$ and the basis defined by the energy levels $\left|n_{a} n_{b}\right\rangle$ of the two oscillators:

$$
\begin{array}{ll}
j=\frac{1}{2}\left(n_{a}+n_{b}\right), & m=\frac{1}{2}\left(n_{a}-n_{b}\right), \\
E|j m\rangle=j|j m\rangle, & J_{z}|j m\rangle=m|j m\rangle .
\end{array}
$$

Each particle $\pi_{i}$ can therefore be seen as arising from a pair of harmonic oscillators $\left(a_{i}, b_{i}\right)$ with total energy fixed to $j$. Thus, we seek for the algebra of operators constructed from pairs of harmonic oscillators, with fixed energy, and invariant under the global $S U(2)$ action. It is given by [42] single-particle operators

$$
E_{i}=\frac{1}{2}\left(a_{i}^{\dagger} a_{i}+b_{i}^{\dagger} b_{i}\right)
$$

and operators acting on each pair of spins

$$
\begin{aligned}
& E_{i j}=\frac{1}{2}\left(a_{i}^{\dagger} a_{j}+a_{j}^{\dagger} a_{i}+b_{i}^{\dagger} b_{j}+b_{j}^{\dagger} b_{i}\right), \\
& F_{i j}=\frac{i}{2}\left(a_{i}^{\dagger} a_{j}-a_{j}^{\dagger} a_{i}+b_{i}^{\dagger} b_{j}-b_{j}^{\dagger} b_{i}\right) .
\end{aligned}
$$

This way, we obtain $N+2 N(N-1) / 2=N^{2}$ operators. It is straightforward to check that the $E_{i}, E_{i j}, F_{i j}$ are all Hermitian operators and form a $u(N)$ Lie algebra. Then, quotienting by the trivially invariant operator $E=\sum_{i} E_{i}$, we get that the invariant algebra is $s u(N)$. It is useful to introduce the following operators $G_{i j}=E_{i j}+i F_{i j}$ and $G_{i j}^{\dagger}=E_{i j}-i F_{i j}=G_{j i}$, which have the following commutation relations:

$$
\begin{gathered}
{\left[G_{i j}, G_{i j}^{\dagger}\right]=2\left(E_{j}-E_{i}\right), \quad\left[G_{i j}, E_{i}\right]=+\frac{1}{2} G_{i j},} \\
{\left[G_{i j}, E_{j}\right]=-\frac{1}{2} G_{i j}^{\dagger}, \quad\left[G_{i j}, G_{k i}\right]=G_{k j} .}
\end{gathered}
$$

The quantum relational coordinates for particle $k$ can be expressed in terms of these operators

$$
\mathfrak{p}_{\alpha}=\frac{1}{2} G^{\dagger k \alpha} G^{k \alpha}-E^{k} E^{\alpha}-E^{k} .
$$

As a final comment, note that this "spin particles universe" can be interpreted as an intertwiner and associated to a fuzzy geometry [42].
[1] G. Amelino-Camelia, Mod. Phys. Lett. A 17, 899 (2002).

[2] U. Harbach, B. Koch, M. Bleicher, H. Stoecker, and S. Hossenfelder, Int. J. Mod. Phys. 20, 3334 (2005).

[3] S. C. Zhang, T. H. Hansson, and S. Kivelson, Phys. Rev. Lett. 62, 82 (1989).
[4] G. Amelino-Camelia, Int. J. Mod. Phys. D 11, 35 (2002).

[5] G. Amelino-Camelina, Nature (London) 418, 34 (2002).

[6] J. Kowalski-Glikman, Lect. Notes Phys. 669, 131 (2005).

[7] J. Kowalski-Glikman, arXiv:gr-qc/0603022.

[8] S. Liberati, S. Sonego, and M. Visser, Phys. Rev. D 71, 
045001 (2005).

[9] R. Aloisio, A. Galante, A. Girillo, S. Liberati, E. Luzio, and F. Méndez, Phys. Rev. D 73, 045020 (2006).

[10] R. Aloisio, A. Galante, A. F. Grillo, S. Liberati, E. Luzio, and F. Mendez, Phys. Rev. D 74, 085017 (2006).

[11] C. Rovelli, Phys. Rev. D 65, 124013 (2002).

[12] C. Rovelli, Quantum Gravity (Cambridge University Press, Cambridge, UK, 2004).

[13] S. B. Giddings, D. Marolf, and J. B. Hartle, Phys. Rev. D 74, 064018 (2006).

[14] L. Smolin, Nucl. Phys. B742, 142 (2006).

[15] Y. Aharonov and L. Susskind, Phys. Rev. 155, 1428 (1967).

[16] Y. Aharonov and T. Kaufherr, Phys. Rev. D 30, 368 (1984).

[17] C. Rovelli, Classical Quantum Gravity 8, 297 (1991).

[18] G. J. Milburn, Phys. Rev. A 44, 5401 (1991).

[19] A. Kitaev, D. Mayers, and J. Preskill, Phys. Rev. A 69, 052326 (2004).

[20] D. Poulin, Int. J. Theor. Phys. 45, 1189 (2006).

[21] A. Peres and P. F. Scudo, Phys. Rev. Lett. 86, 4160 (2001).

[22] A. Peres and P.F. Scudo, Phys. Rev. Lett. 87, 167901 (2001).

[23] S. D. Bartlett, T. Rudolph, and R. W. Spekkens, Phys. Rev. A 70, 032321 (2004).

[24] R. Gambini, R. Porto, and J. Pullin, New J. Phys. 6, 45 (2004).
[25] S. D. Bartlett, T. Rudolph, R.W. Spekkens, and P. S. Turner, New J. Phys. 8, 58 (2006).

[26] S. D. Bartlett, T. Rudolph, and R. W. Spekkens, Rev. Mod. Phys. 79, 555 (2007).

[27] D. Poulin and J. Yard, New J. Phys. 9, 156 (2007).

[28] S. Majid and H. Ruegg, Phys. Lett. B 334, 348 (1994).

[29] H. S. Snyder, Phys. Rev. 71, 38 (1947).

[30] J. Magueijo and L. Smolin, Phys. Rev. D 67, 044017 (2003).

[31] S. Peremelov, J. Math. Phys. (N.Y.) 26, 222 (1972).

[32] E. Wigner, Z. Phys. 131, 101 (1952).

[33] H. Araki and M. Yanase, Phys. Rev. 120, 622 (1960).

[34] K. Kraus, Fundamental Notions of Quantum Theory (Academic Press, Berlin, 1983).

[35] W. H. Zurek, Rev. Mod. Phys. 75, 715 (2003).

[36] J.-C. Boileau, L. Sheridan, M. Laforest, and S. Bartlett, J. Math. Phys. (N.Y.) 49, 032105 (2008).

[37] V. Giovannetti, S. Lloyd, and L. Maccone, Science 306, 1330 (2004).

[38] R. Renner, Nature Phys. 3, 645 (2007).

[39] R. Blume-Kohout, H. Ng, D. Poulin, and L. Viola, arXiv:0705.4282.

[40] H. Barnum and E. Knill, J. Math. Phys. (N.Y.) 43, 2097 (2002).

[41] F. Girelli and E. R. Livine, arXiv:gr-qc/0412004.

[42] F. Girelli and E. R. Livine, Classical Quantum Gravity 22, 3295 (2005). 